\title{
Decanulación en Paciente Pediátrica con Enfermedad Neuromuscular: reporte de caso
}

\author{
Decannulation in a Pediatric Patient with Neuromuscular Disease \\ Gloria Concepción Giménez $\mathbb{1}^{1}$, Estefanía Maidana ${ }^{1}$, Shirley Galeano ${ }^{1}{ }^{1}$, Débora Núñez $\mathbb{D}^{1}$, \\ Francisco Prado Antlagic $\mathbb{D}^{2}$, John Bach $\mathbb{D}^{3}$
}

${ }^{1}$ Universidad Nacional de Asunción, Facultad de Ciencias Médicas, San Lorenzo, Paraguay.

${ }^{2}$ Hospital Clínico San Borja Arriarán, Santiago, Chile

3University Hospital, Newark, NJ, Estados Unidos

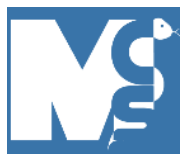

Recibido: 22/11/2020

Revisado: 01/12/2020

Aceptado:07/01/2021

\section{Autor correspondiente}

Gloria Concepción Giménez Universidad Nacional de Asunción, Paraguay

kinesioequipocientifico@gmail.com

\section{Conflictos de interés}

Los autores declaran no poseer conflictos de interés.

\section{Fuente de financiación}

Los autores no recibieron apoyo financiero para la investigación, autoría y/o publicación de este artículo.

Este artículo es publicado bajo una licencia de Creative Commons Reconocimiento 4.0 Internacional.

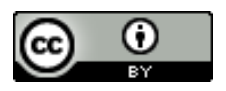

\section{RESUMEN}

La enfermedad de multiminicores es un trastorno neuromuscular hereditario caracterizado por la presencia de múltiples 'cores' en biopsia muscular y características clínicas de una miopatía congénita. El presente caso trata de una paciente de 10 años de edad, con diagnóstico de enfermedad neuromuscular multiminicores, traqueostomizada desde los 7 años de edad por destete fallido y debilidad muscular. La paciente fue derivada al Departamento de Rehabilitación Cardio-respiratoria del hospital de Clínicas de la Facultad de Ciencias Médicas de la Universidad Nacional de Asunción, presentando en su primera evaluación dependencia crónica de oxigeno (por más de 12 meses), tos débil y no funcional con flujo pico tosido e inferior a $160 \mathrm{~L} / \mathrm{m}$, insuficiencia respiratoria crónica e hipercapnia (53 $\mathrm{mmHg}$ de CO2ET). Durante su seguimiento en nuestro departamento fueron seguidas las pautas de Rehabilitación Respiratoria contempladas en el Proyecto de evaluación, tratamiento y seguimiento de pacientes con Enfermedades Neuromusculares, y aprobado por el Consejo Superior de la Facultad de Ciencias Médicas de la Universidad Nacional de Asunción, dichas pautas están fundamentadas en los estudios y publicaciones científicas del Dr. John Bach (Rudgers University, Newart, Nueva Jersey-EEUU) y su equipo colaborador del Grupo Iberoamericano de Cuidados Respiratorios en Enfermedades Neuromusculares. Como resultado final del tratamiento y seguimiento aplicado por más de un año la paciente fue decanulada exitosamente, en un consultorio para pacientes ambulatorios, sin descompensaciones hemodinámicas, con una excelente tolerancia y sin requerimiento de internaciones hospitalarias.

Palabras clave: Enfermedad Neuromuscular; Traqueotomía; Rehabilitación Respiratoria; Decanulación; Enfermedad Multicores.

\section{ABSTRACT}

Multiminicores disease is a hereditary neuromuscular disorder characterized by the presence of multiple 'nuclei' on muscle biopsy and clinical features of a congenital myopathy. The present case concerns a 10year-old patient, diagnosed with multiminicores disease, tracheostomized since she was seven due to failed weaning and muscle weakness. The patient was referred to the Department of Cardio-respiratory Rehabilitation of the Clínicas Hospital from the National University of Asunción, presenting in her first evaluation chronic oxygen dependence (for more than 12 months), weak and non-functional cough with cough peak flow less than $160 \mathrm{~L} / \mathrm{m}$, chronic respiratory failure and hypercapnia $(52 \mathrm{mmHg} C O 2 \mathrm{ET}$ ). We did the follow-up in order to the Respiratory Rehabilitation guidelines contemplated in the Project for the evaluation, treatment and follow-up of patients with Neuromuscular Diseases, which was approved by the Superior Council of the Medical Sciences School from the National University of Asunción, these guidelines are based on scientific studies and publications done by Dr. John Bach (Rudgers University, Newart, New Jersey-USA) and his collaborating team from the Ibero-American Group for Respiratory Care in Neuromuscular Diseases. As a result, the patient was successfully decannulated, in an outpatient clinic, without hemodynamic decompensations, with excellent tolerance and without the requirement of hospital admissions.

Keywords: Neuromuscular Disease; Tracheotomy; Respiratory Rehabilitation; Decannulation; Multicores Disease. 


\section{INTRODUCCIÓN}

La enfermedad de multiminicores ( $\mathrm{MmD}$ ) es un trastorno neuromuscular hereditario caracterizado por la presencia de múltiples 'cores' en biopsia muscular y características clínicas de una miopatía congénita. La acusada variabilidad clínica corresponde a una heterogeneidad genética: el fenotipo clásico más fácilmente reconocible se caracteriza por rigidez espinal, escoliosis prematura y afectación respiratoria, se debe a mutaciones recesivas en el gen de la selenoproteína $\mathrm{N}$ (SEPN1), mientras que las mutaciones recesivas en el receptor de ryanodina del músculo esquelético (RYR1) se asocian a un amplio rango de características clínicas (1).

Los pacientes con enfermedades neuromusculares (ENM) congénitas o adquiridas presentan progresivamente un deterioro de la función respiratoria y, si no es tratada, nos lleva a una tasa de morbilidad, siendo la insuficiencia respiratoria la responsable del evento terminal (2). La detección precoz del compromiso respiratorio y el soporte ventilatorio adecuado permiten una mejor sobrevida y calidad de vida en muchos pacientes (3).

Los pacientes sin ENM que están intubados son extubados según a prueba de respiración espontánea y los parámetros de destete del ventilador. Sin embargo, los pacientes con ENM al ser intubados no cumplen estos parámetros de destete por no poseer la fuerza necesaria para respirar o toser, es por eso que son traqueostomizados $(4,5)$.

A continuación, se presenta el caso clínico de una paciente pediátrica con diagnóstico de $\mathrm{MmD}$, traqueostomizada por más de 3 años, dependiente de oxígeno y con insuficiencia respiratoria crónica quién recibió tratamiento a través del Proyecto de evaluación, tratamiento y seguimiento de pacientes con Enfermedades Neuromusculares, ejecutado por en el Departamento de Rehabilitación Cardio-respiratoria del Hospital de Clínicas.

\section{PRESENTACIÓN DEL CASO}

Escolar, de sexo femenino, con 10 años de edad con diagnóstico médico de miopatía multiminicores. Desde los 15 meses de vida presentó múltiples internaciones por exacerbaciones respiratorias, en su mayoría tratados con oxigenoterapia y antibiótico. A los 7 años de edad fue sometida a traqueostomía debido a intubación prolongada y fallos múltiples del destete. Fue dada de alta con oxigenoterapia nocturna y desde los 8 años fue prescripta con binivel de presión en las vías aéreas (BIPAP) nocturno y oxigenoterapia permanente. Posteriormente consultó en un Hospital pediátrico referente de Enfermedades Neuromusculares en Argentina, constatándose Insuficiencia Respiratoria Crónica (IRC), oxígeno dependencia y uso de ventilación nocturna en forma intermitente, fue internada para tratamiento de su IRC $y$, vuelve a Paraguay sin dependencia de oxígeno.

Fue derivada en nuestro hospital, donde recibió atención multidisciplinaria con asesoramiento de expertos internacionales en el tema, siguiendo las pautas de Rehabilitación respiratoria en Enfermedades Neuromusculares (ENM) estudiadas y publicadas por Bach JR y Cols. (Tabla 1). Iniciando su seguimiento por nuestro equipo, donde fue entrenada para el uso del bolseo manual para asistir la tos con la técnica de air stacking, ya que presentaba tos no funcional con flujo pico tosido (FPT) bajo (FPT < $160 \mathrm{l} / \mathrm{m}$ ) y niveles altos de dióxido de carbono espirado $\left(\mathrm{CO}_{2} \mathrm{ET}, 50 \mathrm{mmHg}\right)$, una vez a normalizada la hipercapnia, se inició la oclusión seriada de la traqueostomía según tolerancia, interrumpiéndose el seguimiento por internación hospitalaria debido a un cuadro respiratorio infeccioso grave. Volviendo al seguimiento se constata disminución notoria de la Capacidad Vital, con una pérdida de más de $400 \mathrm{ml}$, en evaluaciones previas presentaba una CV superior a $1000 \mathrm{ml}$, motivo por el cual se insistieron con el entrenamiento de las técnicas de re expansión pulmonar y asistencia de la tos con apoyo del Asistente Mecánico de la tos (AMT) (Tabla 2).

Un año posterior al inicio de su seguimiento por nuestro departamento, con niveles mejorados de FPT y $\mathrm{CV}$, saturación arterial de oxigeno $\left(\mathrm{SaO}_{2}\right)$ normal y $\mathrm{CO}_{2}$ ET fue decanulada en forma exitosa (Figura 1), continuando con ventilación nocturna a través de soporte ventilatorio no invasivo (SVNI) con equipo binivelado de presión y uso diario del AMT, siendo los valores de flujo pico tosido máximo alcanzado de 200 a 250 L/M, con una capacidad vital (CV) de $950 \mathrm{ml}$, presentando valores normales $\mathrm{CO}_{2} \mathrm{ET}$ y $\mathrm{SaO}_{2}$. Posteriormente a la decanulación se sigue insistiendo con el uso del AMT, además del apilamiento de aires, con lo cual va mejorando su CV y manteniendo valores normales $\mathrm{CO}_{2} \mathrm{ET}$ y $\mathrm{SaO}_{2}$ (Tabla 2). 
TABLA 1. PAUTAS DE REHABILITACIÓN RESPIRATORIA APLICADAS

Pautas de la Rehabilitación Respiratoria en ENM

1- Control regular de: $\mathrm{CV}, \mathrm{FPT}, \mathrm{CO}_{2} \mathrm{ET}$ y $\mathrm{SaO}_{2}$.

2- Introducir Técnica de apilamiento de aire ( Air Stacking) con bolseo manual cuando:

la CV es < al $50 \%$ de lo predicho o $\leq$ a $2000 \mathrm{ml}$ en adultos.

Cuando FPT es < a $270 \mathrm{~L} / \mathrm{m}$

3- Introducir Técnica de Tos Asistida Mecánicamente (TAM) cuando FPT es $\leq$ a $160 \mathrm{~L} / \mathrm{m}$.

4- Cuando la $\mathrm{SaO}_{2}$ es < a 95\%: Usar técnicas de apilamiento de aire y TAM hasta normalizar $\mathrm{SaO}_{2} \geq$ a $95 \%$. Si las técnicas anteriores fallan para normalizar $\mathrm{SaO}_{2} \geq$ a $95 \%$ considerar posibilidad de Hipoventilación, Enfermedad Pulmonar, Neumonía o Atelectasia.

5- En el caso de requerimiento de intubación por Enfermedad Pulmonar severa aplicar TAM cada hora hasta normalizar $\mathrm{SaO}_{2} \geq$ a $95 \%$ sin necesidad de oxigeno suplementario con $\mathrm{CO}_{2}$ normal.

6- En presencia de Hipoventilación Alveolar Crónica Sintomática:

Indicar SVNI nocturno.

Progreso gradual a SVNI diurno según requerimientos.

TABLA 2. RESUMEN DE PARÁMETROS EVALUADOS DURANTE EL TRATAMIENTO Y SEGUIMIENTO DE LA PACIENTE.

\begin{tabular}{llllll}
\hline \multicolumn{1}{c}{ Parámetros } & \multicolumn{1}{c}{ 1ra. Eval. } & \multicolumn{1}{c}{ Seguimiento } & Pre.Extub. & \multicolumn{1}{c}{ Post.Extub. } & \multicolumn{1}{c}{ Al año } \\
\hline$\underline{\text { SaO2 }}$ & $97 \%$ & $97 \%$ & $97 \%$ & $95 \%$ & $97 \%$ \\
$\underline{\text { CO2ET }}$ & $52 \mathrm{mmHg}$ & $40 \mathrm{mmHg}$ & $39 \mathrm{mmHg}$ & $42 \mathrm{mmHg}$ & $42 \mathrm{mmHg}$ \\
$\underline{\text { CV sedente }}$ & $1009 \mathrm{ml}$ & $1100 \mathrm{ml}$ & $960 \mathrm{ml}$ & $740 \mathrm{ml}$ & $900 \mathrm{ml}$ \\
$\underline{\text { CV acostada }}$ & $950 \mathrm{ml}$ & $950 \mathrm{ml}$ & $600 \mathrm{ml}$ & $650 \mathrm{ml}$ & $730 \mathrm{ml}$ \\
$\underline{\text { FPT }}$ & $\mathrm{N} / \mathrm{T}$ & $125 \mathrm{~L} / \mathrm{M}$ & $250 \mathrm{~L} / \mathrm{M}$ & $268 \mathrm{~L} / \mathrm{M}$ & $270 \mathrm{~L} / \mathrm{M}$ \\
\hline
\end{tabular}

FIGURA 1. ESQUEMATIZACIÓN DE PAUTAS SEGUIDAS PARA LA DECANULACIÓN EXITOSA EN ENM.

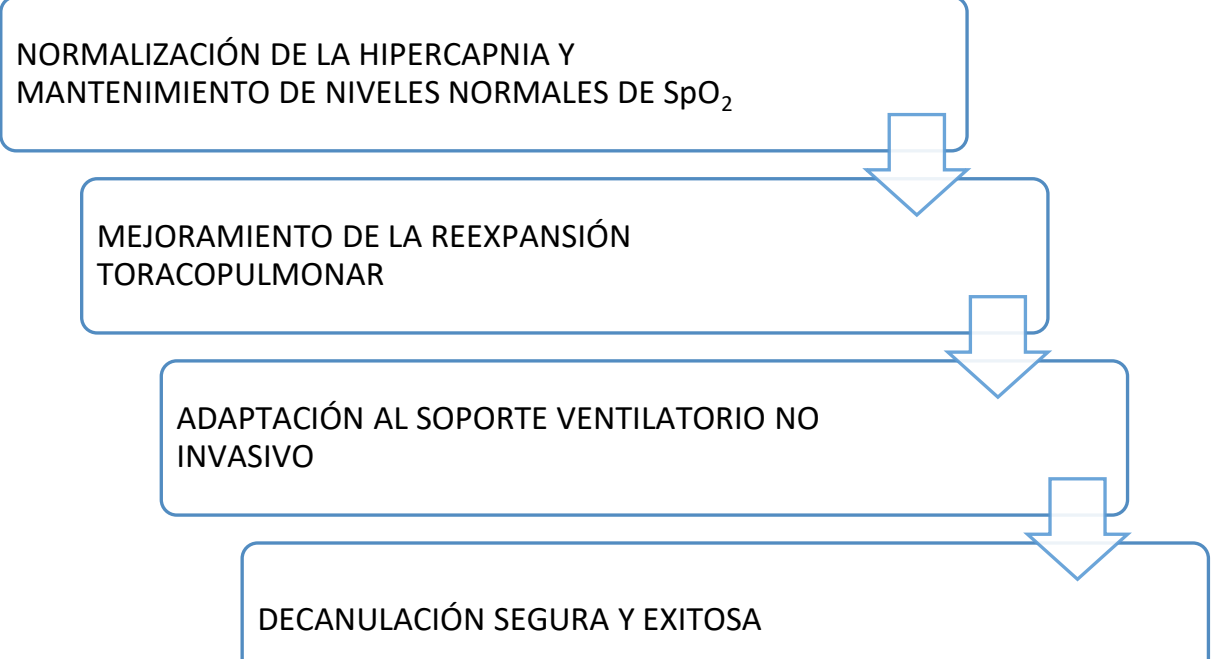

\section{DISCUSIÓN}

El enfoque de tratamiento kinésico enfatizado en el paciente fue en el aparato respiratorio, buscando mantener la compliance toracopulmonar, mantener ventilación pulmonar adecuada, optimizar flujos de la tos para mantener buena permeabilidad de V.A (6), entrenamiento con asistente mecánico de la Tos para promover una decanulación segura $(7,8)$ y entrenamiento con interfaces de VNI (9).

Una evaluación completa y diseñada específicamente para los pacientes con enfermedades neuromusculares especialmente con fallo de la bomba respiratoria puede disminuir e inclusive evitar una IRA y traqueotomía $(10,11)$.
Desafortunadamente cualquier paciente que requiere de servicios respiratorios, sin importar el diagnóstico, que es ingresado a la Unidad de Cuidados intensivos va a requerir de un soporte ventilatorio y una traqueotomía, sin embargo, si es un paciente con ENM se puede optar por procedimiento no invasivos (12), la calidad de vida del paciente se optimiza, así como los gastos hospitalarios.

Los pacientes que llegaron a la intubación podrían ser extubados e incluso decanulados con tratamientos acorde según el tipo de ENM que presenta $(7,8,13)$.

Las terapias de cuidados respiratorios no deben ser postergadas. Para obtener una satisfacción en la funcionalidad ventilatoria, deben ser considerados 
como opción preferente el soporte ventilatorio no invasivo (SVNI) complementado y favorecido por el protocolo de tos asistida manual y mecánicamente, antes de establecer la ventilación mecánica prolongada mediante la traqueotomía. $(14,15)$.

La atención segura y sistematizada cumple una función importante en la recuperación del paciente. La recuperación de un paciente depende de todo un equipo de salud multidisciplinario, siendo la Terapia Respiratoria de enorme importancia clínica, principalmente en patologías donde compromete la funcionalidad (16).

El resultado final obtenido en este caso clínico fue la decanulación con el consecuente mejoramiento de la Calidad de vida. No obstante, como también sucedió con esta paciente, es muy frecuente que, en la historia natural de las enfermedades neuromusculares, incluso en aquellos que pese a su deterioro funcional mantienen la marcha, el compromiso de los músculos respiratorios inspiratorios se traduzca en requerimiento más tardíos de soporte ventilatorio no invasivo por insuficiencia de la bomba respiratoria con hipercapnia progresiva sintomática. Sin embargo, sino se sigue protocolos estandarizados de evaluación, seguimiento e intervención, los niños, adolescentes y adultos con enfermedades neuromusculares son innecesariamente traqueotomizados al momento de presentar infecciones respiratorias agudas altas, que en condiciones de tos insuficiente por compromiso primario de los músculos espiratorios evolucionan a fallo ventilatorio agudo y subsecuentemente a fallo de la extubación al ser intentado el retiro de la ventilación mecánica a ventilación espontánea, sin soporte alguno $(17,18)$.

Estos protocolos, se han sintetizado a nivel latinoamericano en publicaciones del grupo Iberoamericano de cuidados respiratorios en enfermedades neuromusculares $(3,5,6)$ cuyo trabajo ha permitido proyectar la generación de centros de trabajo multidisciplinarios, que se integren a otros a nivel mundial (www.breathenvs.com), con asesoramiento, extensión y capacitación que consideran las pautas fundamentadas en los estudios y publicaciones científicas del Dr. John Bach (Rudgers University, Newart, Nueva Jersey-EEUU) (4,7, 10-12).

La utilidad de esta red de trabajo multidisciplinario permite dar pie para exitosamente retirar la traqueotomía de una paciente con insuficiencia respiratoria crónica hipercapnica avanzada por una miopatía congénita y transformar la dependencia de ventilación mecánica prolongada a necesidad de soporte ventilatorio no invasivo y protocolos de tos asistida, que permiten mejorar las condiciones para la integración familiar, estudiantil, social y posteriormente laboral con oportunidades equivalentes a las de otros personas sin discapacidades físicas. Es así como el objetivo de esta red de centros se basa en ampliar la base conceptual de los profesionales tratantes para comprender que esencialmente ningún paciente con enfermedades neuromusculares independiente de su edad y severidad, excepción hecha, la de aquellos pacientes con esclerosis lateral amiotrofica y otros con compromiso bulbar severo de la primera motoneurona, necesitan traqueostomía para vivir. Comprender que muchos pacientes en estas condiciones clínicas y en cuidados críticos pueden ser extubados a manejos de ventilación no invasiva y tos asistida sin requerir ser traqueostomizados. Reconocer que muchos pacientes traqueostomizados pueden ser decanulados para ser manejados con cuidados respiratorios no invasivos. Reconocer que el escenario natural de estos pacientes debe ser su hogar, con autonomía en la toma de decisiones. Difundir herramientas prácticas para evaluación e implementación del manejo no invasivo de la insuficiencia ventilatoria crónica. Reconocer las diferencias entre ventilación no invasiva y soporte ventilatorio no invasiva y definir los elementos de apoyo diagnóstico y de seguimiento.

\section{REFERENCIAS}

1. Ferreiro A, Quijano-Roy S, Pichereau C, Moghadaszadeh B, Goemans N, Bönnemann C, et al. Mutations of the selenoprotein $\mathrm{N}$ gene, which is implicated in rigid spine muscular dystrophy, cause the classical phenotype of multiminicore disease: reassessing the nosology of earlyonset myopathies. Am J Hum Genet. 2002;71(4):739749. https://doi.org/10.1086/342719r

2. Vega-Briceño L, Contreras I, Prado F, Méndez M, Sánchez I. Evaluación respiratoria de la enfermedad neuromuscular en niños. Neumol pediátr. 2017;2(1):610. URL.

3. Salinas P, Prado F, Pinchak C, Herrero MV, Giménez G, García C, et al. Cuidados respiratorios para pacientes con enfermedades neuromusculares. Neumol Pediatr. 2017;12(3):103-113. URL.

4. Bach JR, Saporito LR. Criteria for extubation and tracheostomy tube removal for patients with ventilatory failure a different approach to weaning. Chest. 1996;110(6):1566-1571.

https://doi.org/10.1378/chest.110.6.1566

5. Pinchak C, Salinas P, Prado F, Herrero M, Giménez G, García C, et al. Actuaización en el manejo respiratorio de pacientes con enfermedades neuromusculares. Arch Pediatr Urug. 2018; 89(1):40-51. http://dx.doi.org/10.31134/ap.89.1.8

6. Giménez G, Prado F, Herrero M, Bach J. Alternativas de tratamiento en pacientes con patologías neuromusculares y afecciones respiratorias. An Fac Cienc Méd. 2017;50(2):79-88. https://doi.org/10.18004/anales/2017.050(02)79-088 
7. Bach JR, Giménez GC, Chiou M. Mechanical InExsufflation-Expiratory Fows as Indication for Tracheostomy Tube Decannuation: Case Studies. Am J Phys Med Rehabil. 2019;98(3):18-20. https://doi.org/10.1097/phm.0000000000000999

8. Torres-Castro R, Monge G, Vera R, Puppo H, Céspedes J, Vilaró J. Esrategias terapéuticas para aumentar la eficacia de la tos en pacientes con enfermedades neuromusculares. Rev méd Chile. 2014;142(2):238-245. http://dx.doi.org/10.4067/S0034-98872014000200013

9. Servera E, Sancho J, Zafra MJ. Tos y enfermedades neuromusculares. Manejo no invasivo de las secreciones respiratorias. Arch Bronconeumol. 2003;39(9):418-427. URL.

10. Bach JR, Alba AS, Saporito LR. Intermittent positive pressure ventilation via the mouth as an aternative to tracheostomy for 257 ventiator users. Chest. 1993;103(1):82-174.

https://doi.org/10.1378/chest.103.1.174

11. Bach JR, Alba AS. Management of chronic alveolar hipoventilation by nasal ventilation. Chest. 1990; 97(1):52-57. https://doi.org/10.1378/chest.97.1.52

12. Bach JR, Niranjan V, Weaver B. Spinal muscular atrophy type 1: A noninvasive respiratory management approach. Chest. $\quad 2000 \quad$ Apr; $117(4): 1100-1105$. https://doi.org/10.1378/chest.117.4.1100

13. Torres R, Kuo CY, Vera R, Espinoza S, Romero JE. Entrenamiento muscular en pacientes traqueotomizados: A propósito de un caso. Neumol pediátr. 2007;5(2):61-63. URL.

14. Elpern EH, Scott MG, Petro L, Ries MH. Pulmonary aspiration in mechanically ventilated patients with tracheostomies. Chest. 1994;105(2):563-566. https://doi.org/10.1378/chest.105.2.563

15. Heffner JE. Timing of tracheotomy in mechanically ventilated patients. Am Rev Respir Dis. 1993;147(3):768771. https://doi.org/10.1164/ajrccm/147.3.768

16. Ishikawa Y. Manual for the Care of Patients Using Noninvasive Ventilation in Neuromuscular disorders. Japan Planning Center Inc., Japan. 2005

17. Servera E, Sancho J, Zafra MJ, Catalá A, Vergara P, Marín J. Alternatives to endotracheal intubation for patients with neuromuscular diseases. Am J Phys Med Rehabil. 2005;84(11):851-857.

https://doi.org/10.1097/01.phm.0000184097.17189.93

18. Servera E, Perez D, Gomez-Merino E, Marin J, Polkey M. Respiratory care units for non-invasive mechanical ventilation in motor neurone disease. Thorax. 2000;55(4):345-346.

http://dx.doi.org/10.1136/thorax.55.4.345a 